\title{
Le mythe de la commune dans les manuels de la première histoire
}

Pierre Guibbert

\section{(2) OpenEdition}

1 Journals

Édition électronique

URL : http://journals.openedition.org/trema/2424

DOI : $10.4000 /$ trema.2424

ISSN : 2107-0997

Éditeur

Faculté d'Éducation de l'université de Montpellier

\section{Édition imprimée}

Date de publication : 1 mai 1992

Pagination : $3-16$

ISSN : 1167-315X

\section{Référence électronique}

Pierre Guibbert, « Le mythe de la commune dans les manuels de la première histoire », Tréma [En ligne], 1 | 1992, mis en ligne le 02 décembre 2013, consulté le 21 avril 2019. URL : http:// journals.openedition.org/trema/2424 ; DOI : 10.4000/trema.2424

Ce document a été généré automatiquement le 21 avril 2019

Trema 


\title{
Le mythe de la commune dans les manuels de la première histoire
}

\author{
Pierre Guibbert
}

1 De nombreux historiographes - de Pierre Nora à Christian Amalvi, en passant par Jacques et Mona Ozouf et Dominique Maingueneau - ont affirmé que la Commune était absente des manuels d'histoire de l'école primaire, ou que ses représentations étaient faibles et rares. Et j'avoue avoir contribué à propager, avec une légèreté coupable, cette thèse dont les implications idéologiques sont évidentes ${ }^{1}$.

2 Je suis revenu sur ce sujet en examinant un échantillon de soixante-trois manuels d'histoire en usage dans l'enseignement primaire français depuis 1893 à nos jours. Un corpus constitué empiriquement mais dont je me suis assuré, après avoir consulté des statistiques de diffusion, qu'il est représentatif des grandes tendances de la production pédagogique française ${ }^{2}$.

\section{La Commune est bien là}

3 Premier constat: la Commune - plus exactement la Commune de Paris ${ }^{3}$ - n'est pas occultée dans les manuels scolaires.

Elle est d'abord présente, pour ainsi dire virtuellement, dans la mesure où elle figure en bonne place dans les Programmes et les Instructions officielles qui orientent l'enseignement de l'histoire à l'école, sinon au niveau du Cours élémentaire - où l'on demande à l'instituteur de passer sans transition de l'exaltation de Gambetta, héros de la Défense nationale, à l'évocation de Jules Ferry, père-fondateur de l'école laïque et de la prospérité coloniale ${ }^{4}$ du moins aux niveaux des Cours moyens et des Classes de Fin d'études.

5 Ainsi, les modèles officiels de répartition définis en 1945 et 1957 proposent-ils que la première semaine du mois de mai, dans la deuxième année du Cours moyen, soit consacrée à deux leçons sur la III République : deux séances couvrant les $2 \mathrm{~h} 30 \mathrm{~min}$ affectées hebdomadairement à l'enseignement de l'histoire, dont la première doit être 
organisée selon le canevas suivant : 1 - La Commune de Paris. 2 - Thiers et la libération du territoire. 3 - Le rapide relèvement de la France. 4 - La Constitution de 1875.

6 Pour donner une échelle de comparaison, la Commune de Paris occupe, dans cette progression, la même place que « Le Régime de Vichy » ou « Louis XVIII et la Charte ».

7 Cependant, plus concrètement, la Commune de Paris est présente dans près de $60 \%$ des manuels considérés ce qui - lorsqu'on entre dans le détail des répartitions niveau par niveau - est conforme aux prescriptions des textes réglementaires.

\section{Une nature indéfinissable}

Quelle fut la nature de la Commune de Paris? Quelles furent les causes de cet événement, et quels en furent les caractères et les péripéties?

9 A ces questions somme toute classiques, qui déterminent souvent l'ordre d'exposition des leçons de la première histoire, les manuels répondent en général sans détours, ce qui est d'un bon rendement pédagogique. Mais ces réponses diffèrent sensiblement selon les convictions et les tempéraments des auteurs et, surtout, selon l'époque de leur rédaction et l'idéologie dominante du temps...

10 A cet égard, on peut distinguer, grosso modo, deux générations de manuels laïques.

11 La première, à laquelle je m'attacherai plus particulièrement, se développe tout au long de la III ${ }^{e}$ République. Elle est le fruit d'une pléiade d'historiens coriaces, qui défie résolument l'ennemi clérical sur son terrain de prédilection. Nombre de manuels confessionnels du temps voient dans la Commune de Paris le syndrome hideux ${ }^{5}$ de la démocratie contemporaine. Les manuels laïques n'éludent pas cette délicate question et produisent, tout à l'avantage de leur cause, leurs propres analyses et leurs propres thèses dont je dois dire, sans parti pris, qu'elles sont en général plus fouillées, plus argumentées et plus cohérentes que celles de l'adversaire juré. C'est d'ailleurs la stabilité de ce discours qui constitue la Commune en objet mythologique plutôt qu'historique ce dont je m'autoriserai, dans un premier temps, pour considérer comme un texte unique ces leçons qui se développent pendant près de cinquante ans...

12 Toutefois cette vigueur idéologique n'exclut pas les tâtonnements théoriques, ce qui peut s'interpréter positivement comme une reconnaissance de l'originalité de la Commune et, négativement, comme un refus d'intégrer l'événement au cours ordinaire de l'Histoire de France.

13 Ainsi, l'on tergiverse lorsqu'il s'agit de définir la nature de la Commune de Paris... S'agit-il d'un Gouvernement révolutionnaire ou d'un simple Conseil municipal? d'un soulèvement, d'une révolte, d'une insurrection ou d'une véritable guerre civile? En la matière, manifestement, les appréciations sont diverses, dès les origines; au point que, confrontés à la précoce richesse de la tradition historiographique, certains auteurs refusent de se prononcer, incapables de définir autrement que par tautologie un événement aussi singulier, qui n'a pas d'antécédent connu: pour beaucoup, la Commune... c'est la Commune.

Relevé lexical portant sur la définition de la nature de l'événement (occurrences classées chronologiquement) : Une guerre civile $(4,7)$ / un gouvernement qui s'intitulait la Commune de Paris (9) / une guerre ouverte contre l'autorité légale (11) / une municipalité qui, sous le titre de Commune, prit le gouvernement de la capitale (13) / un mouvement né sans aucun plan 
d'ensemble et sans idées directrices [qui] resta assez confus quoique, dans son ensemble, de caractère révolutionnaire et socialiste (14) / une nouvelle municipalité qui s'intitula Commune de Paris et qui prétendit se substituer au gouvernement légal du pays (16) / nommé par les Parisiens, un gouvernement révolutionnaire qui s'appela la Commune (18) / sous le nom de Commune, un gouvernement insurrectionnel (19) / 78 membres élus par les quartiers ouvriers, 9 délégués ministres: c'est cette organisation qui s'appelle la Commune (21) / un gouvernement révolutionnaire, la Commune (22) / une insurrection, la Commune (24) / une insurrection (26) / un conseil municipal, la Commune, chargé de gouverner Paris à la place de l'Assemblée installée à Versailles (34) / une insurrection (37) / une guerre civile (41) / une terrible guerre civile, la Commune (43) / le premier gouvernement ouvrier qui ait jamais existé (44) / une révolte [...] un pouvoir révolutionnaire $(45)$ / un gouvernement révolutionnaire $(48,49)$ / un gouvernement à eux [les Parisiens], la Commune (50) / un soulèvement des ouvriers parisiens (57) / un conseil communal élu qui dirige la ville (60) / un gouvernement révolutionnaire (61) / une commune révolutionnaire (62) / une assemblée parisienne élue et disposant de pouvoirs étendus (63).

Cependant, tous les manuels de la première moitié de la III ${ }^{e}$ République se rejoignent pour faire de la Commune, essentiellement, un sous-produit de la guerre de 1870 et, plus précisément, une séquelle du premier siège de Paris. C'est même là leur dénominateur commun. Dans tous ces ouvrages, la cause - à la fois déterminante et conjoncturelle - du déclenchement de la Commune de Paris est la souffrance physique et morale éprouvée par les Parisiens bloqués dans la capitale. Epreuves dont certains manuels - notamment [10,1909 ?, pp. 373 et 375] - nous disent qu'elles ont été catalysées par... la dureté des clauses du Traité de Versailles qui comportent la perspective imminente de l'entrée des Allemands dans Paris.

Et je dois ici ouvrir une rapide parenthèse pour «justifier» cet anachronisme. Ces pédagogues de l'histoire sont, en général, des historiens professionnels. Ils savent que la signature du Traité de Versailles est postérieure au déclenchement de la Commune. Ce qu'ils veulent signifier, par ce raccourci audacieux, c'est que le Peuple de Paris pressent que le prix de la paix serait exorbitant...

Or cette détresse physique et morale est encore amplifiée : par l'inaction - dont les leçons de morale apprenaient alors aux écoliers qu'elle est mauvaise conseillère; par un sentiment de colère à l'encontre d'un gouvernement incapable - et ce sentiment est légitime dès lors que ce gouvernement, qui n'est pas encore républicain, ne saurait être un «bon» gouvernement; et, enfin, par l'amertume qu'engendrent les sacrifices héroïques inutilement consentis - car tous les manuels, dans la leçon précédente, ont crédité le peuple de la capitale d'un comportement exemplaire au cours du premier siège de Paris.

Dans ces conditions, la révolte est inéluctable, quelques formes qu'elle prenne : le peuple de Paris était prêt à toutes les révoltes reconnaissent Gauthier et Deschamps [16, 1923, p. 210]. Il y a là, au fond, je force à peine le trait, une nécessité quasi-physiologique.

Cependant, ces raisons circonstancielles n'excluent pas d'autres mobiles secondaires d'ordre idéologique qui, lorsqu'ils sont évoqués, apparaissent au fond assez superficiels. Certes, les Parisiens sont des Républicains trop ardents, qu'inquiète la composition de la nouvelle Assemblée nationale, en majorité monarchiste. Certes, cette majorité conservatrice multiplie les maladresses en s'installant dans un château de Versailles de fâcheuse mémoire et en supprimant la solde de 1,50F allouée aux Gardes nationaux. Mais on sent bien que ces considérations n'expliquent pas l'événement en profondeur, on devine qu'elles ne rendent compte que de l'anecdote. 


\section{L'inqualifiable} entreprise de caractérisation psychologique de la Commune étayée parfois, exceptionnellement, par des considérations de type sociologique. Ainsi pour Capra [14, 1918, p. 322], l'ensemble des révoltés forme un amalgame détonnant composé :

- de patriotes sincères que nos défaites ont meurtri et exaspéré ;

- de Républicains à principes qui se défient de l'Assemblée nationale ;

- de révolutionnaires exaltés qui prétendent bouleverser la société ;

- et, enfin, de voyous : il y avait à Paris, comme dans toutes les grandes villes, des hommes qui aimaient les violences et les désordres.

Voici pour les origines des meneurs qui entraînent la population de la capitale dans son ensemble ou - selon certains - une partie seulement de cette population. Masses moutonnières, foule à la fois généreuse et veule, magnifique et velléitaire, proie facile des manipulateurs que dénonce férocement un historien, plus engagé que ses confrères, qui ne craint pas de découvrir aux enfants la main d'une Internationale, les agissements des socialistes, Proudhon le Français, Karl Marx l'Allemand, et des anarchistes dont le chef est le Russe Bakounine. [14, 1918, p. 323]. La pré-science du futur, si caractéristique de l'histoire scolaire, débouche parfois, à ce niveau, sur de formidables anticipations qui facilitent la compréhension du présent, comme dans cette légende d'une vignette stipulant : «les otages des communistes, surtout des prêtres, sont fusillés » $(23,1935, \mathrm{p}$. 303).

Voici comment tous ces révoltés finissent par devenir des égarés. Le poids des adjectifs l'atteste, qui diminue l'étendue de leur responsabilité et les disculpe plus qu'il ne les accable : les Communards apparaissent d'abord énervés [21, 1934, p. 224], excités [18, 1934, p. 315], exaspérés [23, 1935, p. 314], ils ont l'esprit troublé [18, 1934, p. 232] et, pour finir, sombrent dans une ultime crise furieuse de folie et de destruction [10, 1909?, p. 373].

C'est en définitive le caractère pathologique de cet ultime paroxysme qui rend compte, a posteriori, de la confusion et de la monstruosité anonymes de la Commune de Paris.

Confusion et inconsistance. Anomalie de l'Histoire, la Commune de Paris est une gigantesque pagaille, un pandémonium, ce qui explique que nos auteurs n'éprouvent pas la nécessité de la raconter. A quoi bon charger la mémoire des écoliers avec des faits qui découragent le «sens de l'Histoire »? A quoi bon leur imposer le récit d'une entreprise qui, à l'évidence, n'a laissé nulle trace dans le présent républicain des enfants de France? Aussi, nulle date ne jalonne cette leçon extraordinaire. Et nulle décision, nulle réalisation de la Commune de Paris n'est évoquée, tant et si bien que la plupart de ces manuels succombent à la tentation d'abréger l'événement en le réduisant à la Semaine sanglante.

Relevé lexical portant sur la qualification de la Commune (occurrences classées chronologiquement): Une terrible guerre civile $(4,7)$ / un lamentable mouvement (9) / une lamentable guerre civile [...] les tristesses d'une épouvantable guerre civile qui ensanglanta la capitale [...] les Français se déchirèrent dans une effroyable guerre civile (11) / une grande honte et de grands désastres [...] la plus criminelle de toutes les insurrections dont l'histoire ait gardé le souvenir (13) / une explosion (14) / horrible bataille fratricide (17) / affreuse guerre de rues [...] un des moments les plus tristes de notre histoire (18) / effroyables combats de rues (22) / 
épouvantable bataille de rues (24) / une terrible insurrection (37) / une atroce guerre civile [...] affreuse guerre civile (43) / une horrible guerre civile (50).

Nul bilan concret, donc. La Commune - je cite ici un jugement parmi les plus pondérés n'eut pas le temps d'appliquer son programme. Elle dut se consacrer tout entière à la lutte contre le gouvernement de Versailles [28, 1943, p. 248]. Au mieux, la Commune de Paris est donc créditée d'intentions non abouties conjuguant, selon les historiens les plus indulgents ou les plus impartiaux, la naïveté et la démesure. Ainsi de Gauthier et Deschamps reconnaissant que ses partisans « avaient rêvé de faire de la France une vaste fédération de Communes libres » $[16,1919$, p. 216].

Quant à la monstruosité de la Commune de Paris, elle est inscrite ontologiquement dans son caractère fratricide que souligne la présence insistante, narquoise, humiliante du regard des Prussiens. La Commune de Paris se déroule, comme un spectacle, «sous les yeux des Prussiens ravis » ainsi que l'écrivaient Gros et Moustiers vers 1905 [8, p. 375]... Ce leitmotiv du regard ennemi est la circonstance aggravante du crime autant que le symptôme de l'égarement des rebelles ${ }^{6}$. Ici, le recours à la redondance apparait comme la figure efficace de la persuasion: «une guerre civile mit aux prises Français contre Français » $[41,1956$, p. 172] ; ou, mieux encore: «il fallut qu'une armée française assiégeât des Français révoltés et prît d'assaut la capitale de la France ». [19, 1932, p. 166].

28 C'est cette folie collective et anonyme - il convient de noter que, dans ces leçons, les Communards n'ont pas de chefs connus, pas de dirigeants nommés ni représentés, et donc pas de héros - qui résout efficacement, au profit des écoliers, le paradoxe historique qui fait naître la $\mathrm{III}^{\mathrm{e}}$ République dans l'opposition à une insurrection somme toute éminemment républicaine.

\section{Le procès}

En tout cas, cet égarement collectif, s'il atténue la responsabilité individuelle des Fédérés, ne justifie pas la Commune de Paris au tribunal de l'Histoire scolaire qui, comme toujours en cas de crise nationale, convoque pour la bonne forme les deux parties antagonistes.

Notons ici que, d'entrée de procès, certains pédagogues se font scrupuleux. «Il est impossible encore d'en faire l'histoire impartiale et exacte» avouent en 1906 les excellents Gauthier et Deschamps [9, p. 302], qui sont à cette époque les best-sellers de l'édition scolaire. Mais cette précaution liminaire, précisément, en établissant celui qui la profère dans les hautes couches de la sérénité, aboutit à donner encore plus de poids à la sentence finale dont il apparaît qu'elle est fondée exclusivement sur des faits patents, avérés - nos juges-historiens nous ont prévenu qu'ils négligeraient les données douteuses - et rendue clans l'impartialité par un juge loyal.

Tout bien pesé, le plateau de la balance penche lourdement au détriment des accusés.

Certes, dans le camp en face, les légalistes ont montré un manque regrettable de sangfroid et d'habileté. Il était maladroit de siéger à Versailles. Il était maladroit de supprimer brutalement la solde des Gardes nationaux. Et puis - Lavisse le reconnaît - les Versaillais fusillaient les prisonniers de guerre...

Cependant, côté Fédérés, que de crimes et d'exactions ! En vérité, prononcent Gauthier et Deschamps dans leurs attendus, «la Commune s'est déconsidérée par d'abominables 
forfaits » $[9,1906$, p. 302] : crimes contre l'humanité - le massacre des otages - et, surtout, crimes contre l'esprit - la destruction du plus prestigieux des patrimoines, le saccage des monuments de Paris, que les auteurs détaillent longuement alors que leurs homologues de l'«Ecole libre " accordent davantage d'attention aux notables civils et religieux assassinés... Ces monuments étaient, littéralement, des reliques sacrées, des morceaux de la vraie Histoire : les historiens scolaires les avaient cités copieusement dans les chapitres précédents comme autant de preuves de l'existence du passé, et ils feront cruellement défaut aux écoliers d'aujourd'hui...

Le triomphe des «méthodes actives" $(12,1914)$ : les élèves de l'Ecole libre pouvaient découvrir par eux-mêmes les crimes des Communards. Ils étaient discrètement aidés par un patient questionnaire destiné à structurer l'observation d'une illustration intitulée «Le massacre des otages »: Pourquoi a-t-on amené ces six hommes contre le mur ? Qui veut les tuer? Pourquoi les Communards vont-ils les fusiller? Lequel est déjà étendu à terre, grièvement blessé? A quoi reconnaissez-vous que c'est le président Bonjean? Comment sont vêtus tous les autres? Que fait le prêtre à genoux, à gauche? Les autres sont-ils déjà blessés? Quelle est leur attitude? Où est l'archevêque de Paris? Que porte-t-il sur la poitrine? Quel geste fait-il en tombant? Pourquoi bénit-il ses ennemis?

Une telle absence de conscience morale et culturelle justifie, rétrospectivement, le châtiment qui s'ensuit.

Cette répression apparaît tout à la fois comme une sainte vengeance - œil pour œil, dent pour dent, c'est l'antique morale immédiate, celle que les enfants réclament et apprécient lorsqu'elle est mise en œuvre à chaud - et, aussi, plus noblement, comme une de ces mémorables expiations publiques, consécutives à une désobéissance grave, qui consacrent la majesté de l'Ordre et de la Loi. Ce qu'expriment les nombreuses formules d'obligation du genre: "Mac Mahon dut faire le siège» ou encore "il fallut qu'une armée française... »:des expressions que les enfants connaissent bien car ils savent que les maîtres châtient les coupables sans plaisir, qu'ils sont obligés de les punir...

Enfin, et peut-être surtout, cette répression est la sanction de l'échec, ce qui est conforme à la rude morale de cette histoire élémentaire qui n'atténue jamais le malheur des vaincus. Malheur mérité puisque ces Communards furent de bien mauvais perdants qui se refusèrent à reconnaître leur infériorité et leur erreur quand il en était encore temps.

\section{La révision}

Les représentations de la Commune de Paris dans les manuels de la III ${ }^{\mathrm{e}}$ République sont donc parfaitement concordantes sur le fond et les différences et les nuances de ton ou de forme, que l'on relève ici et là, sont à mettre au compte d'une option pédagogique particulière ou d'une volonté à s'adapter à un public spécial.

Ainsi certains ouvrages détaillent consciencieusement les différentes sortes de punitions infligées aux Communards: les fusillades, le camp de Satory, l'Afrique, la NouvelleCalédonie, Cayenne... un peu à la manière de certains prêtres du temps qui étaient partisans de ne pas épargner aux jeunes catéchumènes, pour leur édification, la vue des supplices de l'Enfer. Et l'on peut expliquer l'intérêt particulier porté par certains pédagogues « laïques » au sort tragique de monseigneur Darboy-Lavisse est de ceux-là par la volonté commerciale de leurs éditeurs de «mordre" sur la clientèle de l'enseignement privé... 
écis, au fil des éditions successives, on relève une atténuation générale de la des regards et des jugements, voire le développement d'une certaine compassion à l'égard des coupables dont on trouve quelques traces dans les « lectures historiques » qui, dans certains ouvrages, accompagnent l'exposé magistral. Tel manuel reproduit un extrait du Tableau de siège de Théophile Gautier [24, 1935, p. 247]; et, reproduit à l'identique ou dûment "adapté », le célèbre tableau de Danjou, Fusillade au Mur des Fédérés, fournit la matière de plusieurs illustrations.

Le tableau de Danjou, Fusillade au Mur des Fédérés, utilisé par l'illustration scolaire [163, 1981, p. 127].

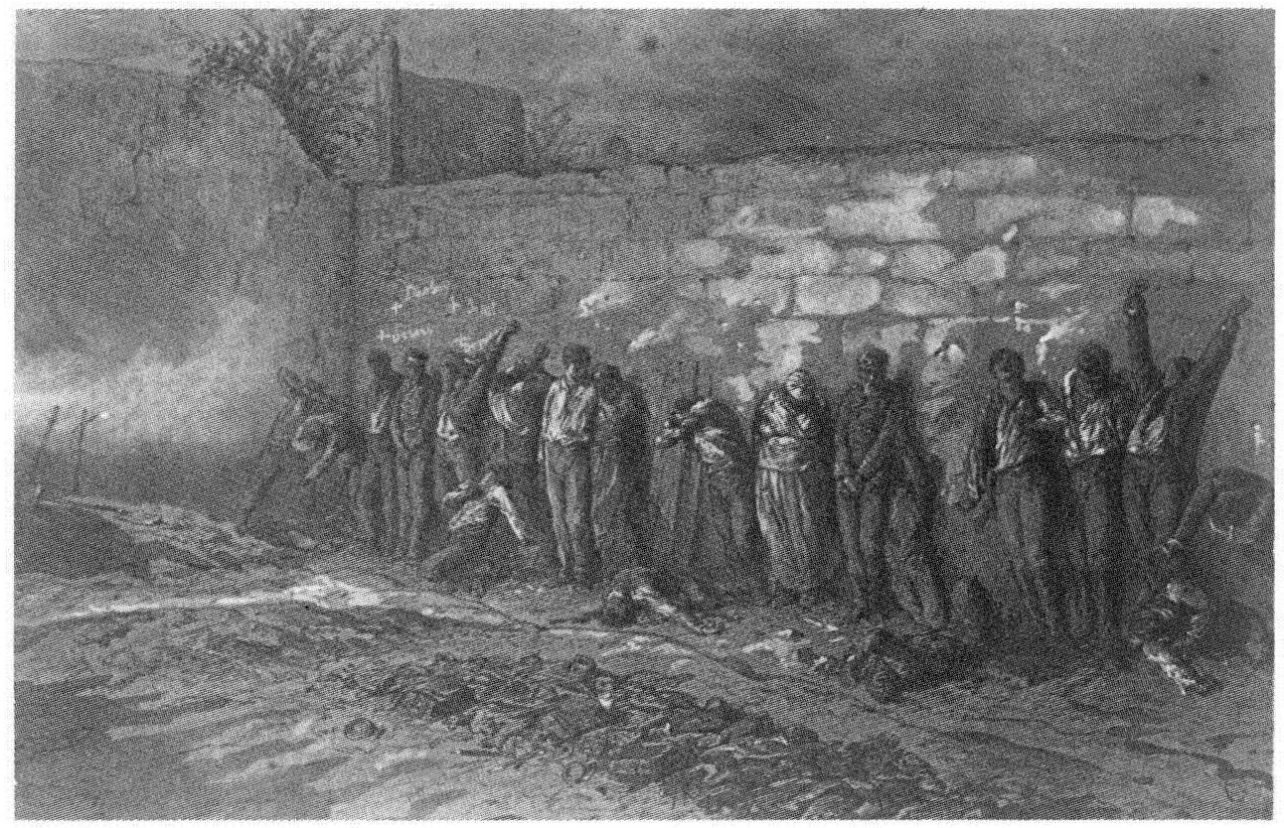

La répression est d'abord qualifiée de terrible [19, p. 166], de sévère [24, 1935, p. 375], puis de brutale [43, 1958, p. 1491, de cruelle [49, 1966, p. 132] ou de très dure [63, 1981, p. 101] et dans ce dernier cas on l'attribue volontiers au seul Mac Mahon, ou à l'armée, de façon à dédouaner Thiers : la figure du père fondateur de la République doit demeurer sans tâche. $\mathrm{Et}$, comme Mac Mahon, bientôt, se révèlera n'être qu'un mauvais Républicain, il s'agit de le discréditer par avance.

Ainsi s'amorce timidement la révision du procès de la Commune de Paris, qu'instruira une deuxième génération d'historiens, postérieurement à la Deuxième guerre mondiale. Ces nouveaux pédagogues établiront la respectabilité de la Commune en la dotant d'une généalogie historique.

Dans ces manuels - dont je ne détaillerai pas les contenus car ils nous sont plus familiers la Commune apparait en amont comme l'héritière directe de la Révolution de 1848 et, plus largement, de la longue série des révoltes parisiennes et nationales qui s'étage de 1830 à 1870 , révoltes dont on indique le caractère social. Complémentairement, en aval, cette nouvelle perspective crédite la Commune, "dernière Révolution de notre histoire ", d'une heureuse postérité : les idées sociales de son programme - dont l'existence est enfin reconnue - seront plus tard réalisées par la République.

Et même, exceptionnellement, on perçoit dans ce concert des voix étrangement discordantes telles celles de Dupaquier et Canac, de véritables «enragés» qui ne 
craignent pas de braver les textes officiels pour jeter, en 1959, à l'usage des enfants du Cours élémentaire, les bases d'une véritable contre-mythologie nationale en saluant dans la Commune le premier pouvoir ouvrier et le germe de notre histoire future [25, 1959, p. 48]...

Toutefois, de telles libertés ne doivent pas nous égarer... Les nouveaux historiens scolaires épousent sagement, à leur manière, les fluctuations des idéologies et l'évolution de l'historiographie "savante». Aussi, globalement, la place qu'ils accordent à la Commune diminue sensiblement, souvent au profit d'événements plus contemporains qui sont censés, peut-être, revêtir les mêmes significations - comme la Révolution russe ou, pour rester dans les limites de l'hexagone, le Front populaire.

D'ailleurs, si le territoire de l'histoire n'en finit pas de se développer, la place impartie à son enseignement a tendance à se réduire...

\section{Le juste milieu}

Pour finir, je voudrais dépasser le simple constat et risquer quelques hypothèses générales sur les raisons et sur le sens de cette présence - ou de cette absence - de la Commune à l'école primaire. Car, ne l'oublions pas, le premier parcours historique de l'enfant, celui du Cours élémentaire, fait l'économie de cet événement...

Serait-ce qu'il n'y eut pas de grands hommes susceptibles d'incarner, au niveau de l'initiation historique, le sens d'un épisode d'essence collective? Mais tous ceux qui ont étudié les mythologies scolaires savent que les pédagogues excellent dans l'art et la manière de susciter les héros : il ne tenait qu'à leur volonté de distinguer une figure - fûtelle de second plan - et de la transfigurer comme ils l'avaient fait, pour d'autres époques, du Grand Ferré ou de Jeanne Hachette. Ainsi, un des rares manuels qui manifeste une sympathie ouverte pour la Commune $[63,1981$, p. 101] a su mettre en scène, dans une posture avantageuse, Louise Michel ${ }^{7}$.

S'agissait-il d'épargner à la sensibilité de tout jeunes enfants le spectacle affreux de la discorde civile et son cortège d'atrocités ? Je ne le crois pas davantage dans la mesure où ces mêmes ouvrages - je pense notamment aux manuels de la III et de la IV République ne répugnent jamais à illustrer la violence et la cruauté, pour peu qu'elles soient éducatives. En témoignent, entre autres, les représentations de la Saint-Barthélémy analysées par Philippe Joutard et Janine Estèbe ${ }^{8}$.

Mais il est vrai qu'ils ne le font jamais gratuitement, et que la Commune offense le sens principal de l'Histoire scolaire. Les troubles de la Commune apparaissent, dans cette histoire téléologique finalisée par la Révolution de 1789, comme une bizarrerie, voire un anachronisme. La présentation de cette résurgence d'un passé archaïque, au niveau élémentaire, risquerait de troubler les très jeunes intelligences encore incapables de distinguer l'accessoire du principal, le nécessaire de l'accidentel.

$51 \mathrm{Au}$ fond, dans cette perspective, la Commune n'est pas tant laide que fausse. Et ce n'est pas tant une faute contre la morale civile qu'une faute politique contre la Nation et contre la République.

52 En dernière instance la Commune de Paris apparait, dans les manuels à l'usage des Cours moyens et des Classes de Fin d'Etudes, comme investie d'une double fonction. 

dont elle aggrave d'ailleurs les conséquences: pour certains auteurs, les rigueurs du Traité de Francfort sont en effet directement imputables à la rébellion. A ce niveau, il semble que cet événement ne soit porteur d'aucun enseignement original pour l'avenir, et qu'il n'est retenu que parce qu'il illustre les conséquences funestes du non respect des enseignements du passé : la Commune de Paris, hyperboliquement, c'est ce qu'il pourrait advenir aux petits Français devenus grands dès lors qu'ils ne retiendraient pas bien les leçons de leur Histoire...

Cependant - et c'est là sa fonction positive - la Commune de Paris contre-balance symétriquement les excès et les troubles que ne vont pas tarder à fomenter les extrémistes de l'autre bord, royalistes et conservateurs - tous ces complots pour renverser la jeune République dont les détails étaient donnés, souvent, dans la foulée de la leçon suivante...

Ici, la représentation de la Commune de Paris prend tout son sens politique. Elle établit la IIIe République naissante dans la légitimité idéale du Souverain Bien dont chacun savait, à cette époque philosophique, qu'il a la particularité d'être hors de l'Histoire. Elle l'établit dans la sphère de l'Idéal, qui brille loin au-dessus de l'horizon historique.

Et, peut-être, certains expliqueront-ils par là, voire justifieront son progressif effacement, rançon de son inutilité contemporaine, en des temps où l'on répète de tous bords que la République est définitivement inscrite dans nos mentalités et que l'Histoire est morte.

\section{BIBLIOGRAPHIE}

Les références bibliographiques abrégées des sources ont été classées chronologiquement. La mise en gras des références numériques indique la présence de la Commune; le sigle * signale les manuels laïques, le sigle $•$ les manuels confessionnels.

01 *Blanchet D. : Histoire de France - $8^{\mathrm{e}}$ (Paris, E. Belin, 1883).

02 *Auge C. et Petit M. : Livre Préparatoire d'Histoire de France (Paris, Larousse, 1893 ?).

03 *Ducoudray G : Histoire et civilisation de la France - C.E. (Paris, Hachette, 1894).

04 *Drouard Ch., MAnNeVy A. : Histoire de France - C.M. (Paris, H. Le Soudier, 1898).

05 *VAST H. et JALliffier R. : Histoire - E.P.S. (Paris, Delagrave, 1901 ?).

06 - GAGNOL abbé : Histoire de France - Premier cours (Paris, Poussielgue, 1902).

07 * Moustier D. : Histoire de France - C.E. (Paris, E. André Fils, 1905).

08 *Gros J., moustiers A. : Histoire de France et Notions d'Histoire générale (Paris, E. André, 1905 ?).

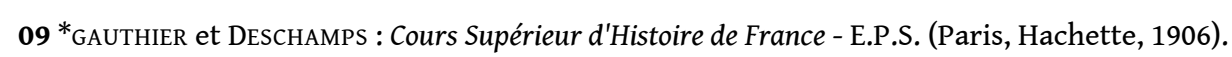

10 •une réunion de professeurs : Histoire de France - C.M. (Mame/Poussielgue, 1909 ?). 
11 *Ammann A. et coutant E.-C : Notions sommaires d'Histoire générale et Révision de l'Histoire de France - E.P.S. (Paris, Nathan, 1910).

12 •GUIRAUD J. : Histoire de France - C.P. (Paris, J. de Gigord, 1914).

13 * LAVISSE E. : La deuxième année d'Histoire de France et d'Histoire générale - C.S. (Paris, Librairie Armand Colin, 1916).

14 *APRA P. : Histoire de France - C.M./F.E. (Paris, Garnier, 1918).

15 * GAUTHIER et Deschamps : Cours Supérieur d'Histoire de France - E.P.S. (Paris, Hachette, 1919).

16 * Gauthier et Deschamps : Cours d'Histoire de France - E.P.S. (Paris, Hachette, 1923).

17 *GAUTHIER et DesChAMPS : Leçons complètes d'Histoire - E.P.S. (Paris, Hachette, Nle éd. 1926).

18 *aVISSE E. : Histoire de France - C.M.1 (Paris, A. Colin, 1934).

19 -SECONDE. : Histoire de France - C.E. (Paris, Hatier, 1932).

20 *Bernard P., REDON F. : Petite Histoire de la France et de la civilisation française (Paris, Nathan, 1934).

21 * BeSSEIGE P., LyONNET A. : Histoire de France - C.M.2 (Paris/Strasbourg, Librairie Istra, 1934).

22 * LAVISSE E. (avec la coll. de conARD P.) : Histoire de France - C.M.2, et F.E. (Paris, A. Colin, 1934).

23. un comité de professeurs : Histoire de France - C.S., progr. du B.E. (Lyon/Paris, E. Vitte, 1935).

24 *Brossolette L : Histoire de France - C.M., F.E. (Paris, Delagrave, 1935).

25 *Brossolette L. : Histoire de France - C.E. (Paris, Delagrave, 1938).

26 *Bougle, Lefranc G. : Histoire du travail et de la civilisation - F.E. (Paris, Sudel, 1938).

27 *Moraze Ch., Henry F. : Histoire du travail et de la civilisation - F.E. (Paris, Hachette, 1939).

28 -FAY B., MAUREL Bl., EQUY J. : Histoire de France Des origines à nos jours - F.E. (Paris, J. de Gigord, 1943).

29 *Audrin E., Dechappe Mme et L. : Notre France son Histoire - C.M. (Paris, Charles-Lavauzelle, 1946).

30 *Personne E., Ballot M., MARC G. : Histoire de France - C.E.1/C.E.2 (Paris, A. Colin, 1949).

31 *Anscombre J. : Cahier de résumés d'Histoire (s. 1., J. Anscombre, 1950).

32 •Billebault E. : Il y avait autrefois... - C.E. (Paris, Les Editions de l'Ecole, 1950 ?).

33 *Lavisse E. : Histoire de France - C.E. (Paris, Librairie Armand Colin, nle éd., 1951).

34 *Personne E., Ballot M., MARC G. : Histoire de France- C.M.1/CM.2 (Paris, A. Colin, 1951).

35 *Belot H. : Histoire de l'Antiquité à nos jours - F.E. (Paris/Strasbourg, Librairie Istra, 1952).

36 * Bonifacio A., MERIeult L. : Histoire de France - C.E. (Paris, Hachette, 1952).

37 * Bonnefin A, MARChAnd M, : Histoire de France et d'Algérie- C.M./C.S (Paris, Hachette, 1953).

38 *AUdRIN Mme, DECHAPPE L. : Notre France, son Histoire - C.E. (Paris, Charles-Lavauzelle \& Cie, 1954).

39 * BERNARd P., REDON F. : Notre premier livre d'Histoire - C.E. (Paris, Nathan, 1954).

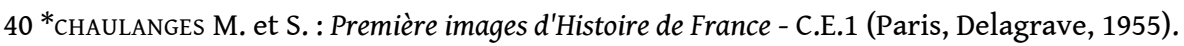


41 * DAVID, FERRE, POITEVIN : Histoire - C.M.1 (Paris, F. Nathan, 1956).

$42{ }^{*}$ Chaulanges M. et S. : Images et récits d'Histoire de France - C.E. (Paris, Delagrave, 1957).

43 * Pradel E., Vincent M. : Histoire de France - C.M.(Paris, S.U.D.E.L., 1958).

44 * CANAC H., DUPAquieR J. : Couleurs de l'Histoire - C.E. (Paris, Didier, 1959).

45 * GRIMAL H., MOREAU L. : Histoire de France - C.M. (Paris, F. Nathan, 1960).

46 * Bonifacio A., Marechal P. : Histoire de France - C.E.1 (Paris, Hachette, 1961).

47 * GRIMAL H., MOREAU L : Histoire de France - C.E. (Paris, F. Nathan, 1962).

$48{ }^{*}$ Chaulanges M. et S. : L'Histoire vivante - F.E. (Paris, Delagrave, 1963).

49 * PeRsonne E., BAllot M., MARC G. : Nouveau livre d'Histoire de France - C.M. (Paris, A. Colin, 1966).

50 * PLANDE R., DECHAPPE M. et L. : Histoire - C.M. (Paris, Charles-Lavauzelle, 1966).

$51{ }^{*}$ AUDRIN Mme, DECHAPPE L. : Histoire de France, images et récits - C.E. (Paris, Charles-Lavauzelle \& Cie, 1968).

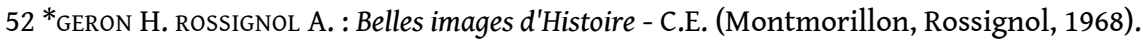

53 * Personne E., ballot M., marc G. : Nouveau livre d'Histoire de France - CE. (Paris, A. Colin, 1968).

54 *aGEORGES J. et ANSCOMBRE J. : Images et récits d'Histoire - C.E. (Paris, M.D.I., 1969).

55 * Pradel E., VincEnt M. : Histoire de France - C.E. (Paris, S.U.D.E.L., 1973).

56 * BABIN J. et alia : Documents et Civilisation (Paris, Hachette, 1974).

57 * BEGUE S., CIAIS R., MEUlEAU M. : La France et les Français autrefois - C.M. (Paris, Bordas, 1976).

58 * Combes J., BUZACoux A. : Histoire - CE. (Paris, Nathan, 1977).

59 * Drouet J.P, Martinez Y., hay R. : Du Passé vers l'avenir- C.M. (Paris, Magnard, 1980).

60 * BiscarRat H. et collectif : Histoire de France - C.M. (Paris, Delagrave, 1981).

61 * Dorel-FerRe G. (avec la coll. de DHAinAut L., Lesiak S.) : Histoire - C.M. (Paris, A. Colin, 1981).

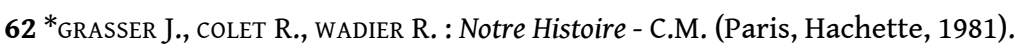

63 * HinNeWinkel. M.J., HinNEWINKEL. J.C., SiviRnE J.M., VinCENT M. : Histoire - C.M. (Paris, F. Nathan, 1981).

\section{NOTES}

1. cf. notamment Claude BILLARD et Pierre GUIBBERT: Histoire mythologique des français (Paris, Galilée, 1976), « L'Age mythologique » (in H Histoire n 1, Paris, Hachette, 1979, pp. 81-98), « Une machine à fabriquer l'Histoire " (in Historiens et Géographes n² 284, Paris, 1981, pp. 833-845) et «Peut-on encore enseigner l'histoire aux enfants?» (in Le Débat, Paris, Gallimard, 1981, pp. 84-95).

2. Un chercheur reconnu comme Christian AMALVI avait documenté son étude sur Les Héros de l'Histoire de France (Paris, Phot'œil, 1979, 315 p.) par un corpus de 60 ouvrages.

3. Je n'ai trouvé aucune allusion aux Communes "provinciales» dans les manuels de l'école primaire. 
4. Cette restriction explique vraisemblablement la cécité des historiographes, qui ont en général privilégié les manuels de la première histoire...

5. J'emprunte l'épithète à l'abbé Gagnol : « La Commune est une hideuse collection d'ennemis de toute autorité, de toute religion, de toute propriété.» $(6,1902$, p. 307). La première référence numérique renvoie à l'ordre de présentation des sources.

6. Cette image a la vie dure : LAVISSE reprend la même formule en 1934 [22, p. 315] et M. et S. CHAUlAnges, en 1955, évoquent encore «le maréchal [qui] assiégea Paris sous les yeux des Allemands ravis de nos discordes [42, p. 375]. Les enfants retrouvaient ce thème dans les manuels de lectures de la III ${ }^{e}$ République. Les Contes du Lundi d'Alphonse DAUDET, notamment, développaient ce motif dans La Mort de Chauvin et dans L'Enfant espion.

7. En regard de ce tableau dramatique reconstituant l'arrestation de Louise Michel figure un extrait de ses Mémoiressous le titre «Louise Michel devant ses juges".

8. Philippe JOUTARD et Janine ESTEBE: La Saint-Barthélémy ou les résonnances d'un massacre (Neuchâtel, Delachaux \& Niestlé, 1976, 245 p.).

\section{RÉSUMÉS}

Dans les manuels scolaires, la Commune apparaît comme la dernière révolution de l'Histoire de France. Révolution laide et fausse, politiquement et moralement, car elle divise les Français sous les yeux de l'ennemi héréditaire. Révolution inutile, puisque la IIIe ${ }^{\circ}$ République est là. Le tableau de ses «excès », la discrédite et sa répression assied la légitimité du nouveau régime.

In scholar handbooks, the Commune seems to be the last revolution in the history of France. It was a false revolution in a political and moral sense since it divides the French Nation under the eyes of the hereditary enemy. An unnecessary revolution since the Third Republic bas already been founded. The list of its «excesses», the discredit and its repression establish the legitimacy of the new regime.

\section{INDEX}

Keywords : commune, history, myth, primary school, school textbook

Mots-clés : commune, enseignement primaire, histoire, manuels scolaires, mythe

\section{AUTEUR}

PIERRE GUIBBERT

Professeur de français, IUFM de Montpellier 